Background Robots are increasingly being used to support management in certain areas of healthcare education. However, the potential application of robotics in palliative care education or simulation has not been explored.

Aim This collaborative project between Computer Science and Palliative Care aimed to program a robot to convey emotion in response to human interaction, in order to develop a robotics program for potential use in palliative care education. Methods The Nao robot is an autonomous, programmable humanoid robot that is controlled by a Linux-based operating system. The robot has capabilities for voice recognition and sound localisation (in-built microphones), multilingual text-tospeech synthesis (in-built speakers) and vision, which include facial and shape recognition (in-built high definition cameras). The robot was programmed by a computer scientist to convey ten emotions (relaxed, anger, withdrawn/sad, lightly crying, heavy sobbing, happy/excited, scared, tired, laughing and dancing) through its posture, movement and speech, in response to human-voiced questions and interaction.

Results The robot was successfully programmed to convey the ten target emotions in response to direct questions posed by a human subject. Discussions around the robot's displayed emotions were explored (e.g. "why are you sad?") to assess the potential of human-computer interaction. The robot continues to acquire a growing lexicon of vocabulary, in addition to an increasing number actions and responses. The robot acts both autonomously and through direct instruction of the operator. Conclusions We have successfully programmed a robot to interact with humans and display emotional responses. This technology could potentially be used to develop innovative ways to engage individuals in discussion about palliative care issues and create opportunities to use robots for interactive educational activity. Consequently, further research can explore the potential to use robotic technology in palliative care for education, and to promote discussion with the public (e.g. children) and healthcare professionals.

\section{0-8 IN SILICO MODELLING OF THE PLASMA MORPHINE CONCENTRATION AND THE LEGAL DRIVING LIMIT}

${ }^{1}$ Jason W Boland, ${ }^{1}$ Miriam J Johnson, ${ }^{2}$ David J Berry. ${ }^{1}$ Hull York Medical School, University of Hull, Hull, UK; ${ }^{2}$ School of Medicine, Pharmacy and Health, Durham University, Durham, UK

\subsection{6/bmjspcare-2017-00133.8}

Background The legal blood morphine concentration for driving in England and Wales has been set at $80 \mu \mathrm{g} / \mathrm{L}$, based on consensus. There is little information regarding the doses likely to cause levels above this limit. Knowledge of the doseconcentration relationship would aid doctors' prescribing decisions and individualised advice. The aim of this study was to investigate the in silico relationship of oral morphine dose and plasma concentration in the context of a morphine plasma concentration of $80 \mu \mathrm{g} / \mathrm{L}$ in different patient groups.

Methods A dose-concentration in silico model for different genders, ages and morphine formulations was generated using Simcyp, a population-based pharmacokinetic simulator. This software simulates absorption and metabolism in a physiology based modelling platform, calculating a range of steady state dose-plasma concentrations across a diverse population. The morphine model created was validated against clinical pharmacokinetic data for oral immediate-release and modifiedrelease preparations. This model calculates only morphine concentrations not its active metabolites; in line with the driving law.

Results Older age, female gender, modified-release formulation and renal dysfunction were associated with higher plasma concentrations at steady state. Except in females over 80 years old or in people with impaired renal function, morphine doses below $120 \mathrm{mg} / \mathrm{d}$ were unlikely to result in a morphine plasma concentration above $80 \mu \mathrm{g} / \mathrm{L}$. In males less than 40 years old with normal renal function, doses up to $250 \mathrm{mg} / \mathrm{d}$ were unlikely to result in a morphine plasma concentration above $80 \mu \mathrm{g} / \mathrm{L}$. An immediate-release morphine dose taken alongside modified-release morphine leads to a higher plasma concentration.

Conclusion These derived morphine dose-concentrations could provide a reference frame for the prescribing clinician. However, the decision and communication to the patient must primarily take into account clinical judgment, the individual patient's level of impairment and insight for any given dose and plasma concentration.

\section{0-9 PHASE 3 STUDY TO EVALUATE THE EFFICACY AND SAFETY OF NALDEMEDINE FOR THE TREATMENT OF OPIOID-INDUCED CONSTIPATION (OIC) IN CANCER PATIENTS}

${ }^{1}$ Takaaki Yokota, ${ }^{2}$ Nobuyuki Katakami, ${ }^{3}$ Toshiyuki Harada, ${ }^{1}$ Yukio Tada, ${ }^{4}$ Masaru Narabayashi, ${ }^{5}$ Narikazu Boku. ${ }^{1}$ Shionogi and Co., Ltd., Osaka, Japan; ${ }^{2}$ Institute of Biomedical Research and Innovation, Kobe, Japan; ${ }^{3} \mathrm{~J}$ CHO Hokkaido Hospital, Sapporo, Japan; ${ }^{4}$ Cancer Institute Hospital of JFCR, Tokyo, Japan; ${ }^{5}$ National Cancer Centre Hospital, Tokyo, Japan

\subsection{6/bmjspcare-2017-00133.9}

Background While opioid analgesics play a central role in managing cancer pain, opioid-induced constipation (OIC) is one of the most common side effects. Naldemedine is a peripherally-acting $\mu$-opioid receptor antagonist being developed to treat OIC.

Methods Studies consisted of a 2 week randomised doubleblind placebo-controlled treatment period (DBT) followed by a 12 week open-label extension (EXT). In DBT, cancer patients with OIC, defined as $\leq 5$ spontaneous bowel movements (SBMs) 14 day before randomization, were randomised 1:1 to oral naldemedine $0.2 \mathrm{mg}$ QD or placebo. Patients who completed DBT could receive naldemedine in EXT. The primary endpoint of DBT was SBM responder rate (percentage of patients with $\geq 3 \mathrm{SBMs} /$ week and an increase from baseline of $\geq 1 \mathrm{SBM} /$ week) in the naldemedine group compared with placebo. The primary objective of EXT was to assess longterm safety.

Results A total of 193 patients were randomised in DBT, and 131 patients were enrolled in EXT. In DBT, significantly higher SBM responder rate was observed in naldemedine compared with placebo $(71.1 \%$ vs $34.4 \%$, respectively; $\mathrm{p}<0.0001)$. Naldemedine improved change from baseline in the frequency of SBMs (5.16 vs 1.54, p<0.0001), SBMs with a feeling of complete evacuation $(2.76$ vs $0.71, \mathrm{p}<0.0001)$ and SBMs without straining (3.85 vs $1.17, \mathrm{p}=0.0005)$ per week. Incidences of adverse event (AE) reported during treatment period in DBT were $44.3 \%$ and $26.0 \%$ in naldemedine and placebo, 